\title{
WEAK AND STRONG CONVERGENCE THEOREMS FOR FIXED POINTS OF GENERALIZED $\alpha$-NONEXPANSIVE MAPPINGS WITH AN APPLICATION
}

\author{
UNWANA E. UDOFIA ${ }^{1}$, AUSTINE E. OFEM $^{2, *}$, AND DONATUS I. IGBOKWE ${ }^{3}$
}

\begin{abstract}
The purpose of this article is to establish weak and strong convergence results of AI iterative scheme for fixed points of generalized $\alpha$-nonexpanisve mappings in uniformly convex Banach spaces. Furthermore, we carry out a numerical experiment to compare the convergence of AI iterative scheme with several prominent iterative schemes. Finally, we use AI iteration process to find the unique solution of a functional Volterra-Fredholm integral equation with deviating argument in Banach spaces. The results of this paper are new and extend several results in the literature.
\end{abstract}

\section{INTRODUCTION}

Let $K$ be a nonempty closed convex subset of a Banach space $B$. By a fixed point of a self mapping $V: K \rightarrow K$, we mean a point $\psi \in K$ which satisfies $V \psi=\psi$. Throughout this paper, $F(V)$ will denote the set of all fixed point of $V, \Re$ will stand for the set of real numbers. The map $V$ is called a contraction if a constant $\delta \in(0,1)$ exists such that $\|V \psi-V \eta\| \leq \delta\|\psi-\eta\|$, for all $\psi, \eta \in K$. When $\delta=1$, then $V$ is a nonexpansive mapping (i.e., $\|V \psi-V \eta\| \leq\|\psi-\eta\|$ ). The mapping $V$ is said to be quasi-nonexpansive if $F(V) \neq \emptyset$ such that $\|V \psi-z\| \leq\|\psi-z\|$, for all $z \in F(V)$ and $\psi \in K$.

Consider a fixed point iteration which is given by

$$
\psi_{s+1}=V \psi_{s}, \forall s \geq 1 \text {. }
$$

The iterative method is known as Picard iteration or the method of successive substitution.

In 1922, a Polish mathematician by name S. Banach in [7] established the metric fixed point theorem for contraction mappings. The theorem is also known as Banach contraction principle. In the case where we have a nonexpansive mapping, it is well known that the Banach contraction principle does not hold since the Picard iteration method fails to converge to the fixed point of nonexpansive mappings, even when the existence of a fixed point of $V$ is guaranteed. For example, let $V:[0,1] \rightarrow[0,1]$ be defined by $V(\psi)=1-\psi$, for all $\psi \in[0,1]$. Then it is clear

\footnotetext{
${ }^{1}$ Department of Mathematics, Akwa Ibom State University, Ikot Akpaden, Nkpatenin, Nigeria

${ }^{2}$ Department of Mathematics, University of Uyo, Uyo, Nigeria

${ }^{3}$ Department of Mathematics, Michael Okpara University of Agriculture, Umudike, Nigeria

*Corresponding aUthor

E-mail addresses: unwanaudofia.aksu@yahoo.com, of emaustine@gmail.com, igbokwedi@yahoo.com.

Key words and phrases. Banach space; generalized $\alpha$-nonexpansive mapping; weak and strong convergence;

AI iterative scheme; Volterra Fredholm integral equation.
}

Received 03/08/2021. 
that $V$ is a nonexpansive mapping with a unique fixed point as $\frac{1}{2}$. If we take an initial value $\psi_{1} \neq \frac{1}{2}$, then successive iterations of $V$ yield the sequence $\left\{1-\psi_{1}, \psi_{1}, 1-\psi_{1}, \ldots\right\}$.

In 1965, F. E. Browder, D. Gohde and W. A. Kirk [10,16,24] showed that every nonexpansive mapping defined on a bounded closed convex subset of a uniformly convex Banach space (or more generally reflexive Banach space having normal structure) has at least a fixed point. Their works were the foundation of the fixed point theory for nonexpansive mappings which illustrated the primary role of the geometry of Banach spaces in this axis of research. It was the birth of an interesting domain of nonlinear functional analysis which attracted the attention of many mathematicians. For more details, see [4, 8, 13-15].

Motivated by the above results, several authors have established different generalizations of nonexpansive mappings.

In [38], Suzuki showed that the class of Suzuki generalized nonexpansive mapping is more general than the class of nonexpansive mapping and obtain some fixed points and convergence theorems. Suzuki generalized nonexpansive mapping is also known as mapping satisfying condition (C).

Definition 1.1. A mapping $V: K \rightarrow K$ is said to be a Suzuki generalized nonexpansive mapping if for all $\psi, \eta \in K$, we have

$$
\frac{1}{2}\|\psi-V \psi\| \leq\|\psi-\eta\| \Longrightarrow\|V \psi-V \eta\| \leq\|\psi-\eta\| .
$$

The class of $\alpha$-nonexpansive mapping was introduced in 2011 by Aoyama and Kohsaka [6] as generalization of nonexpansive mapping and obtained some convergence results.

Definition 1.2. A mapping $V: K \rightarrow K$ is said to be $\alpha$-nonexpansive if there exists $\alpha \in[0,1)$ such that

$$
\|V \psi-V \eta\|^{2} \leq \alpha\|V \psi-\eta\|^{2}+\alpha\|V \eta-\psi\|^{2}+(1-2 \alpha)\|\psi-\eta\|^{2},
$$

for all $\psi, \eta \in K$.

It is worthy to note that nonexpansive mappings are continuous on their domains, but Suzukitype generalized nonexpansivemappings and $\alpha$-nonexpansivemappings need not be continuous (see [38]). Clearly, every nonexpansive mapping is an $\alpha$-nonexpansive mapping with $\alpha=0$ (i.e., 0-nonexpansive) and every $\alpha$-nonexpansive mapping with a nonempty fixed point set is quasi-nonexpansive.

In [31], Pant and Shukla introduced a more general class of nonexpansive mappings in Banach spaces known as generalized $\alpha$-nonexpansive mapping which contains the class of Suzuki generalized nonexpansive mappings.

Definition 1.3. A mapping $V: K \rightarrow K$ is said to be generalized $\alpha$-nonexpansive if there exists $\alpha \in[0,1)$ such that

$$
\begin{aligned}
& \frac{1}{2}\|\psi-V \psi\| \leq\|\psi-\eta\| \text { implies } \\
& \|V \psi-V \eta\| \leq \alpha\|V \psi-\eta\|+\alpha\|V \eta-\psi\|+(1-2 \alpha)\|\psi-\eta\|
\end{aligned}
$$

for all $\psi, \eta \in K$. 
This class of mapping has recently received massive attentions from several authors (see for example, [20,31,34,45] and the references therein).

In the last 2 decades, so many authors have introduced new iteration processes to approximate the fixed points of various classes of mappings. Some of these iteration processes are: Mann iteration [26], Ishikawa iteration [21], Noor iteration [30], Argawal et al. iteration [3], Abbas and Nazir iteration [2], SP iteration [33], S* iteration [22], CR iteration [12], Normal-S iteration [35], Picard-S iteration [18], Thakur iteration [40], Thakur New iteration [41], M iteration [43], Garodia and Uddin [17], M* iteration [42] and so on.

In 2007, the following iterative scheme which is known as S iteration was introduced by Argawal et al. [3]:

$$
\left\{\begin{array}{l}
\psi_{0} \in K \\
\eta_{s}=\left(1-\delta_{s}\right) \psi_{s}+\delta_{s} V \psi_{s}, \\
\psi_{s+1}=\left(1-\beta_{s}\right) V \psi_{s}+\beta_{s} V \eta_{s},
\end{array} \quad \forall s \geq 1 .\right.
$$

where $\left\{\delta_{s}\right\}$ and $\left\{\beta_{s}\right\}$ are sequences in $(0,1)$.

In 2013, Khan [23] introduced the following iterative scheme which is known as normal-S iteration scheme:

$$
\left\{\begin{array}{l}
\psi_{0} \in K \\
\eta_{s}=\left(1-\beta_{s}\right) \psi_{s}+\beta_{s} V \psi_{s}, \quad \forall s \geq 1 . \\
\psi_{s+1}=V \eta_{s},
\end{array}\right.
$$

where $\left\{\beta_{s}\right\}$ is sequence in $(0,1)$. The author showed that normal-S iteration process (1.3) converges at a rate faster than all of Picard, Mann, Ishikawa iteration processes for contraction mappings.

In 2014, the following iterative method known as Picard-S iteration was introduced by Gursoy and Karakaya [18]:

$$
\left\{\begin{array}{l}
\psi_{0} \in K \\
k_{s}=\left(1-\delta_{s}\right) \psi_{s}+\delta_{s} V \psi_{s}, \\
\eta_{s}=\left(1-\beta_{s}\right) V \psi_{s}+\beta_{s} V k_{s}, \quad \forall s \geq 1 \\
\psi_{s+1}=V \eta_{s}
\end{array}\right.
$$

where $\left\{\delta_{s}\right\}$ and $\left\{\beta_{s}\right\}$ are sequences in $(0,1)$. The authors showed with the aid of an example that Picard-S iteration process (1.4) converges at a rate faster than all of Picard, Mann, Ishikawa, Noor, SP, CR, S, S*, Abbas and Nazir, Normal-S and Two-Step Mann iteration processes for contraction mappings.

In 2016, Thakur et al. [41] introduced the following three steps iterative scheme:

$$
\left\{\begin{array}{l}
\psi_{0} \in K \\
k_{s}=\left(1-\delta_{s}\right) \psi_{s}+\delta_{s} V \psi_{s}, \\
\eta_{s}=V\left(\left(1-\beta_{s}\right) \psi_{s}+\beta_{s} k_{s}\right), \quad \forall s \geq 1 . \\
\psi_{s+1}=V \eta_{s}
\end{array}\right.
$$

where $\left\{\delta_{s}\right\}$ and $\left\{\beta_{s}\right\}$ are sequences in $(0,1)$. With the help of numerical example they proved that (1.5) is faster than Picard, Mann, Ishikawa, Agarwal, Noor and Abbas iteration process for suzuki generalized nonexpansive mappings. 
In 2018, Ullah and Arshad [43] introduced the following three steps iterative scheme known as $\mathrm{M}$ iteration process:

$$
\left\{\begin{array}{l}
\psi_{0} \in K, \\
k_{s}=\left(1-\beta_{n}\right) \psi_{s}+\beta_{s} V \psi_{s}, \quad \forall s \geq 1 ; \\
\eta_{s}=V k_{s}, \\
\psi_{s+1}=V \eta_{s},
\end{array}\right.
$$

where $\left\{\beta_{s}\right\}$ is a sequence in $(0,1)$. Numerically, they showed that M iteration process (1.6) converges faster than S iteration process (1.2) and Picard-S iteration process (1.4) for Suzuki generalized nonexpansive mapping. Also, they noted that the speed of convergence of Picard-S iteration process (1.4) and Thakur New iteration (1.5) are almost same.

Recently, Ullah [45] showed that M iteration process (1.6) enjoyed better rate of convergence than Man, Ishikawa, Picard-S, S iteration processes for generalized $\alpha$-nonexpansive mappings.

In 2020, Ofem and Igbokwe [32] introduced the following four steps iterative method known as AI iteration process:

$$
\left\{\begin{array}{l}
\psi_{0} \in K \\
g_{s}=\left(1-\beta_{s}\right) \psi_{s}+\beta_{s} V \psi_{s}, \\
k_{s}=V g_{s}, \\
\eta_{s}=V k_{s}, \\
\psi_{s+1}=V \eta_{s},
\end{array} \quad \forall s \geq 1 .\right.
$$

where $\left\{\beta_{s}\right\}$ is a sequence in $(0,1)$.

The authors proved analytically and numerically that AI iterative method (1.7) has a better speed of convergence than M, Picard-S, Normal-S and Garodia and Uddin iteration processes for contraction mappings. It should be noted here that $M$ iteration process (1.6) and AI iteration process (1.7) are independent.

Motivated by the above results, in this paper, we extend the result of Ofem and Igbokwe [32] from contraction mappings to generalized $\alpha$-nonexpansive mappings. We provide an example of a generalized $\alpha$-nonexpansive mapping and carry out a numerical experiment with the provided example to show that AI iteration process (1.7) converges faster than a number of existing iterative schemes. Finally, we use AI iteration process (1.7) to find the unique solution of a functional Volterra-Fredholm integral equation with deviating argument in Banach spaces.

\section{Preliminaries}

The following definitions, propositions and lemmas will be useful in proving our main results.

Definition 2.1. A Banach space $B$ is said to be uniformly convex if for each $\epsilon \in(0,2]$, there exists $\delta>0$ such that for $\psi, \eta \in B$ satisfying $\|\psi\| \leq 1,\|\eta\| \leq 1$ and $\|\psi-\eta\|>\epsilon$, we have $\left\|\frac{\psi+\eta}{2}\right\|<1-\delta$.

Definition 2.2. A Banach space $B$ is said to satisfy Opial's condition if for any sequence $\left\{\psi_{s}\right\}$ in $B$ which converges weakly to $\psi \in B$ implies

$$
\limsup _{s \rightarrow \infty}\left\|\psi_{s}-\eta\right\|<\limsup _{s \rightarrow \infty}\left\|\psi_{s}-\eta\right\|, \forall \eta \in B \text { with } \eta \neq \psi .
$$


Definition 2.3. Let $\left\{\psi_{s}\right\}$ be a bounded sequence in $B$. For $\psi \in K \subset B$, we put

$$
r\left(\psi,\left\{\psi_{s}\right\}\right)=\limsup _{s \rightarrow \infty}\left\|\psi_{s}-\psi\right\|
$$

The asymptotic radius of $\left\{\psi_{s}\right\}$ relative to $K$ is defined by

$$
r\left(K,\left\{\psi_{s}\right\}\right)=\inf \left\{r\left(\psi,\left\{\psi_{s}\right\}\right): \psi \in K\right\}
$$

The asymptotic center of $\left\{\psi_{s}\right\}$ relative to $K$ is given as:

$$
A\left(K,\left\{\psi_{s}\right\}\right)=\left\{\psi \in: r\left(\psi,\left\{\psi_{s}\right\}\right)=r\left(K,\left\{\psi_{s}\right\}\right)\right\}
$$

In a uniformly convex Banach space, it is well known that $A\left(K,\left\{\psi_{s}\right\}\right)$ consist of exactly one point.

Definition 2.4. [37] A mapping $V: K \rightarrow K$ is said to satisfy condition $(I)$ if a nondecreasing function $f:[0, \infty) \rightarrow[0, \infty)$ exists with $f(0)=0$ and for all $r>0$ then $f(r)>0$ such that $\|\psi-V \psi\| \geq f(d(\psi, F(V))))$ for all $\psi \in K$, where $d(\psi, F(V))=\inf _{z \in F(V)}\|\psi-z\|$.

Proposition 2.5. [31] Let $K$ be a nonempty subset of a Banach space B. Suppose $V: K \rightarrow K$ is any mapping. Then

(i) If $V$ is a Suzuki generalized nonexpansive mapping, it follows that $V$ is a generalized $\alpha$-nonexpansive mapping.

(ii) Every generalized $\alpha$-nonexpansive mapping with a nonempty fixed point set is quasinonexpansive mapping.

(ii) If $V$ is a generalized $\alpha$-nonexpansive mapping, then $F(V)$ is closed. Moreover, if $B$ is strictly convex and $K$ is convex, then $F(V)$ is also convex.

(iv) If $V$ is a generalized $\alpha$-nonexpansive mapping, then the following inequality holds:

$$
\|\psi-V \eta\| \leq\left(\frac{3+\alpha}{1-\alpha}\right)\|\psi-V \psi\|+\|\psi-\eta\|, \forall \psi, \eta \in K
$$

Lemma 2.6. [31] Let $V$ be a self mapping on a subset $K$ of a Banach space $B$ which satisfies Opial's condition. Suppose $V$ is a generalized $\alpha$-nonexpansive mapping. If $\left\{\psi_{s}\right\}$ converges weakly to $z$ and $\lim _{s \rightarrow \infty}\left\|V \psi_{s}-\psi_{s}\right\|=0$, then $V z=z$. That is, $I-V$ is demiclosed at zero.

Lemma 2.7. [36] Suppose $B$ is a uniformly convex Banach space and $\left\{\iota_{s}\right\}$ is any sequence satisfying $0<p \leq \iota_{s} \leq q<1$ for all $s \geq 1$. Suppose $\left\{\psi_{s}\right\}$ and $\left\{\eta_{s}\right\}$ are any sequences of $B$ such that $\limsup _{s \rightarrow \infty}\left\|\psi_{s}\right\| \leq x$, $\limsup _{s \rightarrow \infty}\left\|\eta_{s}\right\| \leq x$ and $\limsup _{s \rightarrow \infty}\left\|\iota_{s} \psi_{s}+\left(1-\iota_{s}\right) \eta_{s}\right\|=x$ hold for some $x \geq 0$. Then $\lim _{s \rightarrow \infty}\left\|\psi_{s}-\eta_{s}\right\|=\stackrel{s \rightarrow \infty}{=0}$.

\section{Main Results}

In this section, we prove the weak and strong convergence of AI iteration algorithm (1.7) for generalized $\alpha$-nonexpansive mappings in the framework of uniformly convex Banach spaces.

Firstly, we state and prove the following lemmas which will be useful in obtaining our main results.

Lemma 3.1. Let $B$ be a Banach space and $K$ be a closed convex subset of $B$. Let $V: K \rightarrow K$ be a generalized $\alpha$-nonexpansive mapping with $F(V) \neq \emptyset$. If $\left\{\psi_{s}\right\}$ is the iterative sequence defined by (1.7), then $\lim _{s \rightarrow \infty}\left\|\psi_{s}-z\right\|$ exists for all $z \in F(V)$. 
Proof. Let $z \in F(V)$. By Proposition 2.5(ii), we know that every generalized $\alpha$-nonexpansive mapping with $F(G) \neq \emptyset$ is quasi-nonexpansive mapping. So from (1.7), we have

$$
\begin{aligned}
\left\|g_{s}-z\right\| & =\left\|\left(1-\beta_{s}\right) \psi_{s}+\beta_{s} V \psi_{s}-z\right\| \\
& \leq\left(1-\beta_{s}\right)\left\|\psi_{s}-z\right\|+\beta_{s}\left\|V \psi_{s}-z\right\| \\
& \leq\left(1-\beta_{s}\right)\left\|\psi_{s}-z\right\|+\beta_{s}\left\|\psi_{s}-z\right\| \\
& =\left\|\psi_{s}-z\right\| .
\end{aligned}
$$

Using (1.7) and (3.1), we obtain

$$
\begin{aligned}
\left\|k_{s}-z\right\| & =\left\|V g_{n}-z\right\| \\
& =\left\|g_{s}-z\right\| \leq\left\|\psi_{s}-z\right\| .
\end{aligned}
$$

Again, using (1.7) and (3.2), we get

$$
\begin{aligned}
\left\|\eta_{s}-z\right\| & =\left\|V g_{s}-z\right\| \\
& \leq\left\|g_{s}-z\right\| \\
& \leq\left\|\psi_{s}-z\right\| .
\end{aligned}
$$

Lastly, from (1.7) and (3.3), we have

$$
\begin{aligned}
\left\|\psi_{s+1}-z\right\| & =\left\|V \eta_{s}-z\right\| \\
& \leq\left\|\eta_{s}-z\right\| \\
& \leq\left\|\psi_{s}-z\right\| .
\end{aligned}
$$

This implies that $\left\{\left\|\psi_{s}-z\right\|\right\}$ is bounded and nondecreasing for all $z \in F(V)$. Hence, $\lim _{s \rightarrow \infty}\left\|\psi_{s}-z\right\|$ exists.

Lemma 3.2. Let $B$ be a uniformly convex Banach space and $K$ be a nonempty closed convex subset of $B$. Let $V: K \rightarrow K$ be a generalized $\alpha$-nonexpansive mapping. Suppose $\left\{\psi_{s}\right\}$ is the iterative sequence defined by (1.7). Then, $F(V) \neq \emptyset$ if and only if $\left\{\psi_{s}\right\}$ is bounded and $\lim _{s \rightarrow \infty}\left\|V \psi_{s}-\psi_{s}\right\|=0$.

Proof. Suppose $F(V) \neq \emptyset$ and let $z \in F(V)$. Then, by Lemma 4.1, $\lim _{s \rightarrow \infty}\left\|\psi_{s}-z\right\|$ exists and $\left\{\psi_{s}\right\}$ is bounded. Put

$$
\lim _{s \rightarrow \infty}\left\|\psi_{s}-z\right\|=x
$$

From (3.4) and (3.5), we obtain

$$
\limsup _{s \rightarrow \infty}\left\|g_{s}-z\right\| \leq \limsup _{s \rightarrow \infty}\left\|\psi_{s}-z\right\|=x .
$$

From Proposition 2.5(ii), we know that every generalized $\alpha$-nonexpansive mapping with $F(V) \neq$ $\emptyset$ is quasi-nonexpansive mapping. So that we have

$$
\limsup _{s \rightarrow \infty}\left\|V \psi_{s}-z\right\| \leq \limsup _{s \rightarrow \infty}\left\|\psi_{s}-z\right\|=x .
$$


Again, using (1.7), we get

$$
\begin{aligned}
\left\|\psi_{s+1}-z\right\| & =\left\|V \eta_{s}-z\right\| \\
& \leq\left\|\eta_{s}-z\right\| \\
& =\left\|V k_{s}-z\right\| \\
& \leq\left\|k_{s}-z\right\| \\
& =\left\|V g_{s}-z\right\| \\
& \leq\left\|g_{s}-z\right\| .
\end{aligned}
$$

Therefore, from (3.5), we obtain

$$
x \leq \liminf _{s \rightarrow \infty}\left\|g_{s}-z\right\|
$$

From (3.6) and (3.8) we obtain

$$
\begin{aligned}
x & =\lim _{s \rightarrow \infty}\left\|g_{n}-z\right\| \\
& =\lim _{s \rightarrow \infty}\left\|\left(1-\beta_{s}\right) \psi_{s}+\beta_{s} V \psi_{s}-z\right\| \\
& =\lim _{s \rightarrow \infty}\left\|\left(1-\beta_{s}\right)\left(\psi_{s}-z\right)+\beta_{s}\left(V \psi_{s}-z\right)\right\| \\
& =\lim _{s \rightarrow \infty}\left\|\beta_{s}\left(V \psi_{s}-z\right)+\left(1-\beta_{s}\right)\left(\psi_{s}-z\right)\right\| .
\end{aligned}
$$

From (3.5), (3.7), (3.9) and Lemma 2.14, we obtain

$$
\lim _{s \rightarrow \infty}\left\|V \psi_{s}-\psi_{s}\right\|=0 .
$$

Conversely, assume that $\left\{\psi_{s}\right\}$ is bounded and $\lim _{s \rightarrow \infty}\left\|V \psi_{s}-\psi_{s}\right\|=0$. Let $z \in A\left(K,\left\{\psi_{s}\right\}\right)$, by definition 2.3 and Proposition 2.5(iv), we have

$$
\begin{aligned}
\left(V z,\left\{\psi_{s}\right\}\right) & =\limsup _{s \rightarrow \infty}\left\|\psi_{s}-V z\right\| \\
& \leq \limsup _{s \rightarrow \infty}\left(\frac{(3+\alpha)}{(1-\alpha)}\left\|V \psi_{s}-\psi_{s}\right\|+\left\|\psi_{s}-z\right\|\right) \\
& =\limsup _{s \rightarrow \infty}\left\|\psi_{s}-z\right\| \\
& =r\left(z,\left\{\psi_{s}\right\}\right)
\end{aligned}
$$

This implies that $z \in A\left(K,\left\{\psi_{s}\right\}\right)$. Since $B$ is uniformly convex, $A\left(K,\left\{\psi_{s}\right\}\right)$ is singleton, thus we have $V z=z$.

Theorem 3.3. Let $B, K, V$ be as in Lemma 4.2. Suppose tat B satisfies Opial's condition and $F(V) \neq \emptyset$. Then, the sequence $\left\{\psi_{s}\right\}$ defined by (1.7) converges weakly to a fixed point of $V$.

Proof. Let $z \in F(V)$, then by Lemma 4.1, we have $\lim _{s \rightarrow \infty}\left\|\psi_{s}-z\right\|$ exists. Now we show that $\left\{\psi_{s}\right\}$ has weak sequential limit in $F(V)$. Let $\psi$ and $\eta$ be weak limits of the subsequences $\left\{\psi_{s_{j}}\right\}$ and $\left\{\eta_{s_{k}}\right\}$ of $\left\{\psi_{s}\right\}$ respectively. By Lemma 4.2, we have $\lim _{s \rightarrow \infty}\left\|V \psi_{s}-\psi_{s}\right\|=0$ and from Lemma 2.6, $I-V$ is demiclosed at zero. It follows that $(I-V) \psi=0$ implies $\psi=V \psi$, similarly $V \eta=\eta$. 
Next we show uniqueness. Suppose $\psi \neq \eta$, then by Opial's property, we obtain

$$
\begin{aligned}
\lim _{s \rightarrow \infty}\left\|\psi_{s}-\psi\right\| & =\lim _{s_{j} \rightarrow \infty}\left\|\psi_{s_{j}}-\psi\right\| \\
& <\lim _{s_{j} \rightarrow \infty}\left\|\psi_{s_{j}}-\eta\right\| \\
& =\lim _{s \rightarrow \infty}\left\|\psi_{s}-\eta\right\| \\
& =\lim _{s_{k} \rightarrow \infty}\left\|\psi_{s_{k}}-\eta\right\| \\
& <\lim _{s_{k} \rightarrow \infty}\left\|\psi_{s_{k}}-\psi\right\| \\
& =\lim _{s \rightarrow \infty}\left\|\psi_{s}-\psi\right\|,
\end{aligned}
$$

which is a contradiction, so $\psi=\eta$. Hence, $\left\{\psi_{s}\right\}$ converges weakly to a fixed point of $V$.

Theorem 3.4. Let $B, K, V$ be as in Lemma 4.2. Then, the $\left\{\psi_{s}\right\}$ defined by (1.7) converges strongly to a point of $F(V)$ if and only if $\liminf _{s \rightarrow \infty} d\left(\psi_{s}, F(V)\right)=0$, where $d\left(\psi_{s}, F(V)\right)=\inf \{\| \psi-$ $z \|: z \in F(V)\}$.

Proof. Necessity is obvious. Assume that $\liminf _{s \rightarrow \infty} d\left(\psi_{s}, F(V)\right)=0$. From Lemma 4.1, we have $\lim _{s \rightarrow \infty}\left\|\psi_{s}-z\right\|$ exists for all $z \in F(V)$, it follows that $\liminf _{s \rightarrow \infty} d\left(\psi_{s}, F(V)\right)$ exists. But by hypothesis, $\liminf _{s \rightarrow \infty} d\left(\psi_{s}, F(V)\right)=0$, thus $\lim _{s \rightarrow \infty} d\left(\psi_{s}, F(V)\right)=0$. Next we prove that $\left\{\psi_{s}\right\}$ is a Cauchy sequence in $K$. Since $\liminf _{s \rightarrow \infty} d\left(\psi_{s}, F(V)\right)=0$, then given $\varepsilon>0$, there exists $s_{0} \in \mathbb{N}$ such that, for all $s, n \geq s_{0}$, we have

$$
\begin{aligned}
& d\left(\psi_{s}, F(V)\right) \leq \frac{\epsilon}{2} \\
& d\left(\psi_{n}, F(V)\right) \leq \frac{\epsilon}{2} .
\end{aligned}
$$

Thus, we have

$$
\begin{aligned}
\left\|\psi_{s}-\psi_{n}\right\| & \leq\left\|\psi_{s}-z\right\|+\left\|\psi_{n}-z\right\| \\
& \leq d\left(\psi_{s}, F(V)\right)+d\left(\psi_{n}, F(V)\right) \\
& \leq \frac{\epsilon}{2}+\frac{\epsilon}{2}=\epsilon .
\end{aligned}
$$

Hence $\left\{\psi_{s}\right\}$ is a Cauchy sequence in $K$. Since $K$ is closed, therefore there exists a point $\psi_{1} \in K$ such that $\lim _{s \rightarrow \infty} \psi_{s}=\psi_{1}$. Since $\lim _{s \rightarrow \infty} d\left(\psi_{s}, F(V)\right)=0$, it implies that $\lim _{s \rightarrow \infty} d\left(\psi_{1}, F(V)\right)=0$. Hence, $\psi_{1} \in F(V)$ since $F(V)$ closed.

Theorem 3.5. Let B, $K, V$ be as in Lemma 4.2. If $V$ satisfies condition $(I)$, then the sequence $\left\{\psi_{s}\right\}$ defined by (1.7) converges strongly to a fixed point of $V$.

Proof. We have shown in Lemma 4.2 that

$$
\lim _{s \rightarrow \infty}\left\|V \psi_{s}-\psi_{s}\right\|=0
$$

Using condition (I) in Definition 2.10 and (3.13), we get

$$
\lim _{s \rightarrow \infty} f\left(d\left(\psi_{s}, F(V)\right)\right) \leq \lim _{s \rightarrow \infty}\left\|V \psi_{s}-\psi_{s}\right\|=0
$$

i.e., $\lim _{s \rightarrow \infty} f\left(d\left(\psi_{s}, F(V)\right)\right)=0$. Since $f:[0, \infty) \rightarrow[0, \infty)$ is a nondecreasing function satisfying $f(0)=0, f(r)>0$ for all $r \in(0, \infty)$, we have

$$
\lim _{s \rightarrow \infty} d\left(\psi_{s}, F(V)\right)=0 .
$$


From Theorem 4.5, then sequence $\left\{\psi_{s}\right\}$ converges strongly to a point of $F(V)$.

\section{NUMERICAL EXPERIMENT}

In this section, we provide an example of a mapping which is a generalized $\alpha$-nonexpansive, but not Suzuki generalized nonexpansive mapping. With the aid of the example, we will prove that our new iterative algorithm (1.7) outperform a number of iterative algorithms in the existing literature in terms of speed of convergence.

Example 4.1. Let $B=\Re$ with usual $|\cdot|$ and $K=[0, \infty)$. Let $V: K \rightarrow K$ be defined as:

$$
V \psi= \begin{cases}0, & \text { if } \psi \in\left[0, \frac{3}{4}\right), \\ \frac{2 \psi}{3}, & \text { if } \psi \in\left[\frac{3}{4}, \infty\right) .\end{cases}
$$

Now, we show that $V$ is a generalized $\alpha$-nonexpansive mapping, but not Suzuki generalize. For this, let $\psi=\frac{1}{3}$ and $\eta=\frac{3}{4}$, then

$$
\frac{1}{2}|\psi-V \psi|=\frac{1}{6}<\frac{5}{12}=|\psi-\eta|
$$

But

$$
|V \psi-V \eta|=\frac{2 \eta}{3}=\frac{1}{2}>\frac{5}{12}=|\psi-\eta|
$$

Hence, $V$ is not a Suzuki generalized nonexpansive mapping.

To show that $V$ is a generalized $\alpha$-nonexpansive mapping with $\alpha=\frac{1}{2}$ (i.e., generalized $\frac{1}{2}$ nonexpansive), we consider the following possible cases:

Case (I): When $\psi, \eta \in\left[0, \frac{3}{4}\right)$, we have

$$
\begin{aligned}
\alpha\|V \psi-\eta\|+\alpha\|\psi-V \eta\|+(1-2 \alpha) \mid \psi-\eta \| & =\frac{1}{2}|T \ell-\zeta|+\frac{1}{2}|\ell-T \zeta| \\
& \geq 0=|V \psi-V \eta| .
\end{aligned}
$$

Case (II): When $\psi, \eta \in\left[\frac{3}{4}, \infty\right)$, we obtain

$$
\begin{aligned}
\alpha\|V \psi-\eta\|+\alpha\|\psi-V \eta\|+(1-2 \alpha) \mid \psi-\eta \| & =\frac{1}{2}|V \psi-\eta|+\frac{1}{2}|\psi-V \eta| \\
& =\frac{1}{2}\left|\frac{2 \psi}{3}-\eta\right|+\frac{1}{2}\left|\psi-\frac{2 \eta}{3}\right| \\
& \geq \frac{1}{2}\left|\left(\frac{2 \psi}{3}-\eta\right)+\left(\psi-\frac{2 \eta}{3}\right)\right| \\
& =\frac{5}{6}|\psi-\eta| \\
& >\frac{2}{3}|\psi-\eta|=|V \psi-V \eta| .
\end{aligned}
$$

Case (III): When $\psi \in\left[\frac{3}{4}, \infty\right)$ and $\eta \in\left[0, \frac{3}{4}\right)$, we get

$$
\begin{aligned}
\alpha\|V \psi-\eta\|+\alpha\|\psi-V \eta\|+(1-2 \alpha) \mid \psi-\eta \| & =\frac{1}{2}|V \psi-\eta|+\frac{1}{2}|\psi-V \eta| \\
& =\frac{1}{2}\left|\frac{2 \psi}{3}-\eta\right|+\frac{1}{3}|\psi| \\
& \geq \frac{2}{3}|\psi|=|V \psi-V \eta| .
\end{aligned}
$$


Hence, $V$ is generalized $\alpha$-nonexpansive mapping with $\alpha=\frac{1}{2}$ (i.e., generalized $\frac{1}{2}$-nonexpansive) with $F(V)=\{0\}$.

By using the above example, we will show that our new iteration process (1.7) converges faster than S, Picard S, Tharkur, M and Normal S iteration processes. With the aid of MATLAB (R2015a), we observed that Picard-S and Thakur iteration have almost the same speed of convergence and we obtain the comparison Table 1 and Figure 1 for various iterative schemes with control sequences $\delta_{s}=\beta_{s}=\frac{1}{10}$ and initial guess $\psi_{1}=20$.

TABLE 1. Comparison of speed of convergence of our new iterative scheme with S, Picard-S, M and Normal S iterative schemes.

\begin{tabular}{cccccc}
\hline Step & S & Picard-S & M & Normal S & AI \\
\hline 1 & 20.00000000 & 20.00000000 & 20.00000000 & 20.00000000 & 20.00000000 \\
2 & 13.29660239 & 8.86440159 & 8.61952862 & 12.92929293 & 5.74635241 \\
3 & 8.83998175 & 3.92888078 & 3.71481368 & 8.35833078 & 1.65102830 \\
4 & 5.87708612 & 1.74135885 & 1.60099714 & 5.40336535 & 0.47436952 \\
5 & 3.90726387 & 0.77180521 & 0.68999203 & 3.49308467 & 0.13629472 \\
6 & 2.59766670 & 0.34207957 & 0.29737030 & 2.25815575 & 0.00000000 \\
7 & 1.72700707 & 0.15161653 & 0.12815959 & 1.45981786 & 0.00000000 \\
8 & 1.14816631 & 0.00000000 & 0.00000000 & 0.94372064 & 0.00000000 \\
9 & 0.76333555 & 0.00000000 & 0.00000000 & 0.61008203 & 0.00000000 \\
10 & 0.50748846 & 0.00000000 & 0.00000000 & 0.39439646 & 0.00000000 \\
11 & 0.33739362 & 0.00000000 & 0.00000000 & 0.25496337 & 0.00000000 \\
12 & 0.22430944 & 0.00000000 & 0.00000000 & 0.16482480 & 0.00000000 \\
13 & 0.14912767 & 0.00000000 & 0.00000000 & 0.10655341 & 0.00000000 \\
\hline
\end{tabular}

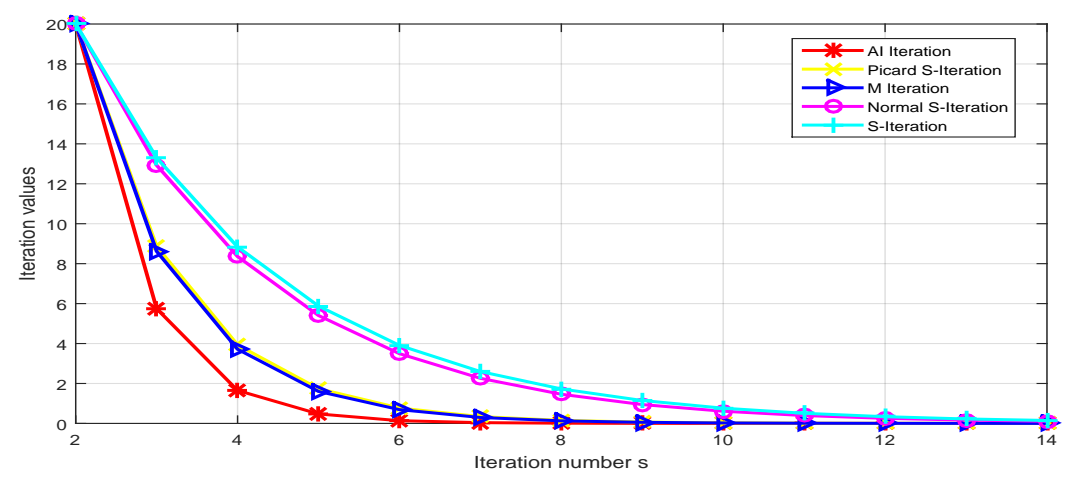

FiguRE 1. Graph corresponding to Table1.

\section{Application}

Several problems which arise in mathematical physics, engineering, biology, economics and etc., lead to mathematical models described by nonlinear integral equations (see [27] and the references therein). In particular, Volterra-Fredholm integral equations arise from parabolic boundary value problems, from the mathematical modeling of the spatio-temporal development of an epidemic, and from various physical and biological models (see [29], [46]). 
In this section, we will use our new iterative method (1.7) to solve the following VolterraFredholm integral equation which have been considered by Lungu and Rus [25]:

$$
u(\psi, \eta)=g(\psi, \eta, h(u(\psi, \eta)))+\int_{0}^{\psi} \int_{0}^{\eta} K(\psi, \eta, m, n, u(m, n)) d m d n
$$

for all $\psi, \eta \in \Re_{+}$. Let Let $(\Omega,|\cdot|)$ be a Banach space. Let $\tau>0$ and

$$
X_{\tau}=\left\{u \in C\left(\Re_{+}^{2}, \Omega\right)|\exists M(u)>0:| u(\psi, \eta) \mid e^{-\tau(\psi+\eta)} \leq M(u)\right\} .
$$

We now consider Bielecki's norm on $X_{\tau}$ as follows:

$$
\|u\|_{\tau}=\sup _{\psi, \eta \in \Re_{+}}\left(|u(\psi, \eta)| e^{-\tau(\psi+\eta)}\right) .
$$

Obviously, $\left(X_{\tau},\|\cdot\|_{\tau}\right)$ is a Banach space (see [9]).

The following result which was given by Lungu and Rus [25] will be useful in proving our main result.

Theorem 5.1. [25] Suppose the following conditions are fulfilled:

$\left(V_{1}\right) g \in C\left(\Re_{+}^{2} \times \Omega, \Omega\right), K \in C\left(\Re_{+}^{4} \times \Omega, \Omega\right) ;$

$\left(V_{2}\right) h: X_{\tau} \rightarrow X_{\tau}$ is such that

$$
\exists l_{h}>0:|h(u(\psi, \eta))-h(v(\psi, \eta))| \leq l_{H}\|u-v\| \cdot e^{\tau(\psi+\eta)},
$$

for all $\psi, \eta \in \Re_{+}$and $u, v \in X_{\tau}$;

$\left(V_{3}\right)$

$$
\exists l_{g}>0:\left|g\left(\psi, \eta, e_{1}\right)-g\left(\psi, \eta, e_{2}\right)\right| \leq l_{g}\left|e_{1}-e_{2}\right|,
$$

for all $\psi, \eta \in \Re_{+}$and $e_{1}, e_{2} \in \Omega$;

$\left(V_{4}\right)$

$\exists l_{K}(\psi, \eta, m, n):\left|K\left(\psi, \eta, m, n, e_{1}\right)-K\left(\psi, \eta, m, n, e_{1}\right)\right| \leq l_{K}(\psi, \eta, m, n)\left|e_{1}-e_{2}\right|$,

for all $\psi, \eta, m, n \in \Re_{+}$and $e_{1}, e_{2} \in \Omega$;

$\left(V_{5}\right) l_{K} \in C\left(\Re_{+}^{4}, \Re_{+}\right)$and

$$
\int_{0}^{\psi} \int_{0}^{\eta} l_{K}(\psi, \eta, m, n) e^{\tau(\psi+\eta)} d m d n \leq l e^{\tau(\psi+\eta)},
$$

for all $\psi, \eta \in \Re_{+}$;

$\left(V_{6}\right) l_{g} l_{h}+l<1$.

Then, the equation (5.1) has a unique solution $z \in X_{\tau}$ and the sequence of successive approximations

$$
u_{s+1}(\psi, \eta)=g\left(\psi, \eta, h\left(u_{s}(\psi, \eta)\right)\right)+\int_{0}^{\psi} \int_{0}^{\eta} K\left(\psi, \eta, m, n, u_{s}(m, n)\right) d m d n,
$$

for all $s \in \mathbb{N}$ converges uniformly to $z$.

We now give our main result in this section.

Theorem 5.2. Let $\left\{\psi_{s}\right\}$ be AI iterative method defined by (1.7) with sequence $\left\{\beta_{s}\right\}$ in [0,1] such that $\sum_{s=0}^{\infty} \beta_{s}=\infty$. If all the conditions $\left(V_{1}\right)-\left(V_{6}\right)$ in theorem 5.1 are satisfied, then the equation (5.1) has a unique solution $z$ in $X_{\tau}$ and the AI iterative sequence (1.7) converges strongly to $z$. 
Proof. Let $\left\{\psi_{s}\right\}$ be an iterative sequence generated by AI iterative method (1.7) for the operator $A: X_{\tau} \rightarrow X_{\tau}$ defined by

$$
A(u(\psi, \eta))=g(\psi, \eta, h(u(\psi, \eta)))+\int_{0}^{\psi} \int_{0}^{\eta} K(\psi, \eta, m, n, u(m, n)) d m d n .
$$

We will prove that $\psi_{s} \rightarrow 0$ as $s \rightarrow \infty$. Using (1.7), we obtain

$$
\left\|\psi_{s+1}-z\right\|_{\tau}=\sup _{\psi, \eta \in \Re_{+}}\left(\left|A\left(\eta_{s}(\psi, \eta)\right)-A(z(\psi, \eta))\right| e^{-\tau(\psi+\eta)}\right) .
$$

Now,

$$
\begin{aligned}
\left.\mid A\left(\eta_{s}(\psi, \eta)\right)\right)- & A(z(\psi, \eta)) \mid \\
\leq & \left|g\left(\psi, \eta, h\left(\eta_{s}(\psi, \eta)\right)\right)-g(\psi, \eta, h(z(\psi, \eta)))\right| \\
& +\mid \int_{0}^{\psi} \int_{0}^{\eta} K\left(\psi, \eta, m, n, \eta_{s}(m, n)\right) d m d n \\
& -\int_{0}^{\psi} \int_{0}^{\eta} K(\psi, \eta, m, n, z(m, n)) d m d n \mid \\
\leq & l_{g}\left|h\left(\eta_{s}(\psi, \eta)\right)-h(z(\psi, \eta))\right| \\
& +\int_{0}^{\psi} \int_{0}^{\eta} \mid K\left(\psi, \eta, m, n, \eta_{s}(m, n)\right) \\
& -K(\psi, \eta, m, n, z(m, n)) \mid d m d n \\
\leq & l_{g} l_{h}\left\|\eta_{s}-z\right\|_{\tau} e^{\tau(\psi+\eta)} \\
& +\int_{0}^{\psi} \int_{0}^{\eta} l_{K}(\psi, \eta, m, n)\left|\eta_{s}(m, n)-z(m, n)\right| d m d s \\
\leq & l_{g} l_{h}\left\|\eta_{s}-z\right\|_{\tau} e^{\tau(\psi+\eta)}+l\left\|\eta_{s}-z\right\|_{\tau} e^{\tau(\psi+\eta)} \\
= & \left(l_{g} l_{h}+l\right)\left\|\eta_{s}-z\right\|_{\tau} e^{\tau(\psi+\eta)} .
\end{aligned}
$$

Hence,

$$
\left\|\psi_{s+1}-z\right\|_{\tau} \leq\left(l_{g} l_{h}+l\right)\left\|\eta_{s}-z\right\|_{\tau}
$$

Also,

$$
\left\|\eta_{s}-z\right\|_{\tau}=\sup _{\psi, \eta \in \Re_{+}}\left(\left|A\left(k_{s}(\psi, \eta)\right)-A(z(\psi, \eta))\right| e^{-\tau(\psi+\eta)}\right) .
$$

Now,

$$
\begin{aligned}
\left.\mid A\left(k_{s}(\psi, \eta)\right)\right)- & A(z(\psi, \eta)) \mid \\
\leq & \left|g\left(\psi, \eta, h\left(k_{s}(\psi, \eta)\right)\right)-g(\psi, \eta, h(z(\psi, \eta)))\right| \\
& +\mid \int_{0}^{\psi} \int_{0}^{\eta} K\left(\psi, \eta, m, n, k_{s}(m, n)\right) d m d n \\
& -\int_{0}^{\psi} \int_{0}^{\eta} K(\psi, \eta, m, n, z(m, n)) d m d n \mid \\
\leq & l_{g}\left|h\left(k_{s}(\psi, \eta)\right)-h(z(\psi, \eta))\right| \\
& +\int_{0}^{\psi} \int_{0}^{\eta} \mid K\left(\psi, \eta, m, n, k_{s}(m, n)\right) \\
& -K(\psi, \eta, m, n, z(m, n)) \mid d m d n
\end{aligned}
$$




$$
\begin{aligned}
\leq & l_{g} l_{h}\left\|k_{s}-z\right\|_{\tau} e^{\tau(\psi+\eta)} \\
& +\int_{0}^{\psi} \int_{0}^{\eta} l_{K}(\psi, \eta, m, n)\left|\eta_{s}(m, n)-z(m, n)\right| d m d s \\
\leq & l_{g} l_{h}\left\|k_{s}-z\right\|_{\tau} e^{\tau(\psi+\eta)}+l\left\|k_{s}-z\right\|_{\tau} e^{\tau(\psi+\eta)} \\
= & \left(l_{g} l_{h}+l\right)\left\|k_{s}-z\right\|_{\tau} e^{\tau(\psi+\eta)} .
\end{aligned}
$$

Hence,

$$
\left\|\eta_{s}-z\right\|_{\tau} \leq\left(l_{g} l_{h}+l\right)\left\|k_{s}-z\right\|_{\tau}
$$

Putting (5.5) into (5.4), we get

$$
\left\|\psi_{s+1}-z\right\|_{\tau} \leq\left(l_{g} l_{g}+l\right)^{2}\left\|k_{s}-z\right\|_{\tau} .
$$

Similarly,

$$
\left\|k_{s}-z\right\|_{\tau} \leq\left(l_{g} l_{h}+l\right)\left\|g_{s}-z\right\|_{\tau}
$$

Putting (5.7) into (5.6), we get

$$
\left\|\psi_{s+1}-z\right\|_{\tau} \leq\left(l_{g} l_{h}+l\right)^{3}\left\|g_{s}-z\right\|_{\tau} .
$$

Again,

$$
\begin{aligned}
\left\|g_{s}-z\right\|_{\tau} & =\left\|\left(\left(1-\beta_{s}\right) \psi_{s}+\beta_{s} A \psi_{s}\right)-z\right\| \\
& =\left\|\left(1-\beta_{s}\right)\left(\psi_{s}-z\right)+\beta_{s}\left(A \psi_{s}-z\right)\right\| \\
& \leq\left(1-\beta_{s}\right)\left\|\psi_{s}-z\right\|+\beta_{s}\left\|A \psi_{s}-z\right\| .
\end{aligned}
$$

Now,

$$
\left\|A \psi_{s}-A z\right\|_{\tau}=\sup _{\psi, \eta \in \Re_{+}}\left(\left|A\left(\psi_{s}(\psi, \eta)\right)-A(z(\psi, \eta))\right| e^{-\tau(\psi+\eta)}\right),
$$

and

$$
\begin{aligned}
\left.\mid A\left(\psi_{s}(\psi, \eta)\right)\right)-A(z(\psi, \eta)) \mid \leq & \left|g\left(\psi, \eta, h\left(\psi_{s}(\psi, \eta)\right)\right)-g(\psi, \eta, h(z(\psi, \eta)))\right| \\
& +\mid \int_{0}^{\psi} \int_{0}^{\eta} K\left(\psi, \eta, m, n, \psi_{s}(m, n)\right) d m d n \\
& -\int_{0}^{\psi} \int_{0}^{\eta} K(\psi, \eta, m, n, z(m, n)) d m d n \mid \\
\leq & l_{g}\left|h\left(\psi_{s}(\psi, \eta)\right)-h(z(\psi, \eta))\right| \\
& +\int_{0}^{\psi} \int_{0}^{\eta} \mid K\left(\psi, \eta, m, n, \psi_{s}(m, n)\right) \\
& -K(\psi, \eta, m, n, z(m, n)) \mid d m d n \\
\leq & l_{g} l_{h}\left\|\psi_{s}-z\right\|_{\tau} e^{\tau(\psi+\eta)} \\
& +\int_{0}^{\psi} \int_{0}^{\eta} l_{K}(\psi, \eta, m, n)\left|\psi_{s}(m, n)-z(m, n)\right| d m d s \\
\leq & l_{g} l_{h}\left\|\psi_{s}-z\right\|_{\tau} e^{\tau(\psi+\eta)}+l\left\|\psi_{s}-z\right\|_{\tau} e^{\tau(\psi+\eta)} \\
= & \left(l_{g} l_{h}+l\right)\left\|\psi_{s}-z\right\|_{\tau} e^{\tau(\psi+\eta)} .
\end{aligned}
$$


Thus,

$$
\left\|A \psi_{s}-A z\right\|_{\tau} \leq\left(l_{g} l_{h}+l\right)\left\|\psi_{s}-z\right\|_{\tau} .
$$

From (5.9) and (5.10), we obtain

$$
\begin{aligned}
\left\|g_{s}-z\right\| & \leq\left(1-\beta_{s}\right)\left\|\psi_{s}-z\right\|+\beta_{s}\left(l_{g} l_{h}+l\right)\left\|\psi_{s}-z\right\|_{\tau} \\
& =\left[1-\beta_{s}\left\{1-\left(l_{g} l_{h}+l\right)\right\}\right]\left\|\psi_{s}-z\right\|_{\tau} .
\end{aligned}
$$

From (5.11) and (5.8), we get

$$
\left\|\psi_{s+1}-z\right\|_{\tau} \leq\left(l_{g} l_{h}+l\right)^{4}\left[1-\beta_{s}\left\{1-\left(l_{g} l_{h}+l\right)\right\}\right]\left\|\psi_{s}-z\right\|_{\tau} .
$$

Recalling from assumption $\left(C_{6}\right)$ that $l_{g} l_{h}+l<1$ and since $\beta_{s} \in[0,1]$, then from (5.12) we have

$$
\left\|\psi_{s+1}-z\right\|_{\tau} \leq\left[1-\beta_{s}\left\{1-\left(l_{g} l_{h}+l\right)\right\}\right]\left\|\psi_{s}-z\right\|_{\tau} .
$$

Inductively, from (5.13), we have

$$
\left\|\psi_{s+1}-z\right\|_{\tau} \leq\left\|\psi_{0}-z\right\|_{\tau} \prod_{k}^{s}\left[1-\beta_{k}\left\{1-\left(l_{g} l_{h}+l\right)\right\}\right] .
$$

Since $\beta_{k} \in[0,1]$ for all $k \in \mathbb{N}$ and assumption $\left(C_{6}\right)$ gives

$$
1-\beta_{k}\left\{1-\left(l_{g} l_{h}+l\right)\right\}<1 .
$$

From classical analysis, we know that $1-\psi \leq e^{-\psi}$ for all $\psi \in[0,1]$. Thus, (5.14) becomes

$$
\left\|\psi_{s+1}-z\right\|_{\tau} \leq\left\|\psi_{0}-z\right\|_{\tau} e^{-\left[1-\beta_{k}\left\{1-\left(l_{g} l_{h}+l\right)\right\}\right] \sum_{k=0}^{s} \beta_{k}}
$$

which yields $\lim _{s \rightarrow \infty}\left\|\psi_{s}-z\right\|_{\tau}=0$. This completes the proof.

\section{Conclusion}

In this article, the weak and strong convergence of AI iterative scheme (1.7) has been studied for a class of mapping which is more general than the class of mapping considered by Ofem and Igbokwe [32]. An example which has been shown to be a generalized $\alpha$-noxpansive mapping has been presented. This example is equally used to compare the rate of convergence of a number of existing iterative schemes and it was confirmed that AI iterative scheme converges faster than M, Normal S, Picard S and S iteration schemes. We also showed that AI iteration converges strongly the unique solution of functional Volterra-Fredholm integral equation with deviating argument. Hence, our results mainly extend the results of Ofem and Igbokwe [32] and several other well known results in the literature.

\section{REFERENCES}

[1] H.A. Abass, A.A. Mebawondu, O.T. Mewomo, Some results for a new three steps iteration scheme in Banach spaces, Bull. Trans. Univ. Brasov, Ser. III: Math. Inform. Phys. 11(60)(2) (2018) 1-18.

[2] M. Abbas, T. Nazir, A new faster iteration process applied to constrained minimization and feasibility problems. Mat. Vesn, 66(2014) 223-234.

[3] R. P. Agarwal, D.O. Regan, D.R. Sahu, Iterative construction of fixed points of nearly asymptotically nonexpansive mappings, J Nonlinear Convex Anal. 8(2007) 61-79.

[4] A.G. Aksoy and M.A. Khamsi, Nonstandard Methods in Fixed Point Theory, Springer-Verlag (1990).

[5] F. Ali, J. Ali and J.J. Nieto, Some observations on generalized non-expansivemappings with an application, Computational and Applied Mathematics, 39(2020) 74 https://doi org/10.1007/s40314-020-1101-4. 
[6] K. Aoyama and F. Kohsaka, Fixed point theorem for $\alpha$-nonexpansive mappings in Banach spaces, Nonlinear Anal. Theory Meth. Appl. 74(13) (2011) 4387-4391. https://doi.org/10.1016/j.na.2011.03.057.

[7] S. Banach, Sur les opérations dans les ensembles abstraits et leur application aux équations intégrales, Fundam. Math. 3 (1922), 133-181.

[8] L.P. Belluce, W.A. Kirk and E.F. Steiner, Normal structure in Banach spaces, Pac. J. Math. 26 (1968) 433-440. https://doi.org/10.2140/pjm.1968.26.433.

[9] A. Bielecki, Une remarque sur l'application de la méthode de Banach-Cocciopoli-Tichonov dans la thórie de l'équation $s=f(x, y, z, p, q)$, Bull. Pol. Acad. Sci. Math. 4 (1956) 265-357.

[10] F.E. Browder, Nonexpansive nonlinear operators in a Banach space, Proc. Nat. Acad. Sci. USA. 54 (1965) 1041-1044. https://doi.org/10.1073/pnas.54.4.1041.

[11] S.K. Chatterjea, Fixed point theorems, C. R. Acad. Bulg. Sci. 25(1972) 727-730.

[12] R. Chugh, V. Kumar and S. Kumar, Strong convergence of a new three step iterative scheme in Banach spaces, Amer. J Comput. Math. 2(2012) 345-357. https://doi.org/10.4236/ajcm.2012.24048.

[13] A. Dehici, S. Atailia, Fixed points of asymptotically nonexpansive mappings with center 0 and applications, ArXiv:2103.10727 [Math]. (2021). http://arxiv.org/abs/2103.10727.

[14] K. Goebel and W. A. Kirk, Topics in metric fixed point theory, Cambridge Studies in Advanced Mathematics. First edition (1990).

[15] K. Goebel, S. Reich, Uniform Convexity, Hyperbolic Geometry, and Nonexpansive Mappings, Marcel Dekker, New York and Basel (1984).

[16] G. Gohde, Zum Prinzip der kontractiven Abbildung, Math. Nach. 30 (1965) 251-258.

[17] [1]C. Garodia, I. Uddin, Solution of a nonlinear integral equation via new fixed point iteration process, ArXiv:1809.03771 [Math]. (2018). http://arxiv.org/abs/1809.03771.

[18] [1]F. Gürsoy, V. Karakaya, A Picard-S hybrid type iteration method for solving a differential equation with retarded argument, ArXiv:1403.2546 [Math]. (2014). http://arxiv.org/abs/1403.2546.

[19] G. Hämmerlin, K.H. Hoffmann, Numerical mathematics. Springer, Berlin (1991).

[20] H. Iqbal, M. Abbas, S. M. Husnine, Existence and approximation of fixed points of multivalued generalized $\alpha$-nonexpansive mappings in Banach spaces, Numer. Algor. 85 (2020) 1029-1049. https://doi.org/10. 1007/s11075-019-00854-z.

[21] S. Ishikawa, Fixed points by a new iteration method. Proc. Amer Math. Soc. 44(1974) 147-150. https: //doi.org/10.1090/S0002-9939-1974-0336469-5

[22] I. Karahan, M. Ozdemir, A general iterative method for approximation of fixed points and their applications, Adv. Fixed Point Theory, 3(2013) 510-526.

[23] [1]S.H. Khan, A Picard-Mann hybrid iterative process, Fixed Point Theory Appl. 2013 (2013) 69. https: //doi.org/10.1186/1687-1812-2013-69.

[24] W. A. Kirk, A fixed point theorem for mappings which do not increase distances, Amer. Math. Mon. 72 (9) (1965) 1004-1006. https://doi.org/10.2307/2313345.

[25] N. Lungu and I.A. Rus, On a functional Volterra-fredholm integral equation, via Picard operators, J. Math. Inequal. 3(4) (2009) 519-527.

[26] W.R. Mann, Mean value methods in iteration, Proc. Amer. Math. Soc. 4(1953) 506-510.

[27] K. Maleknejad, P. Torabi, Application of fixed point method for solving Volterra-Hammerstein integral equation, 74(1) (2012), U.P.B. Sci. Bull. Ser. A. 74(1) (2012) 45-56.

[28] R. Kannan, Some results on fixed point. Bull. Calcutta Math. Soc. 10 (1968) 71-76.

[29] K. Maleknejad, M. Hadizadeh, A New computational method for Volterra-Fredholm integral equations, Comput. Math. Appl. 37 (1999) 1-8. https://doi.org/10.1016/S0898-1221 (99)00107-8.

[30] M.A. Noor, New approximation schemes for general variational inequalities, J. Math. Anal. Appl. 251 (2000) 217-229. https://doi.org/10.1006/jmaa.2000.7042.

[31] D. Pant, R. Shukla, Approximating fixed points of generalized $\alpha$-nonexpansive mappings in Banach spaces, Numer. Funct. Anal. Optim. 38(2) (2017) 248-266.

[32] A. E. Ofem, D. I. Igbokwe, An efficient iterative method and its applications to a nonlinear integral equation and a delay differential equation in Banach spaces, Turk. J. Ineq. 4(2) (2020) 79-107. 
[33] W. Phuengrattana, S. Suantai, On the rate of convergence of Mann, Ishikawa, Noor and SP-iterations for continuous functions on an arbitrary interval, J. Comput. Appl. Math. 235(2011), 3006-3014. https: //doi.org/10.1016/j.cam.2010.12.022.

[34] [1]H. Piri, B. Daraby, S. Rahrovi, M. Ghasemi, Approximating fixed points of generalized $\alpha$-nonexpansive mappings in Banach spaces by new faster iteration process, Numer. Algor. 81 (2019) 1129-1148. https: //doi.org/10.1007/s11075-018-0588-x.

[35] D. R. Sahu, A. Petrusel, Strong convergence of iterative methods by strictly pseudocontractive mappings in Banach spaces. Nonlinear Anal. Theory Meth. Appl. 74 (2011) 6012-6023. https://doi.org/10.1016/ j.na.2011.05.078.

[36] J. Schu, Weak and strong convergence to fixed points of asymptotically nonexpansive mappings, Bull. Aust. Math. Soc. 43 (1991) 153-159. https://doi.org/10.1017/S0004972700028884.

[37] H.F. Senter, W.G. Dotson, Approximating fixed points of nonexpansive mapping, Proc. Amer. Math. Soc. 44(1974) 375-380. https://doi.org/10.1090/S0002-9939-1974-0346608-8.

[38] T. Suzuki, Fixed point theorems and convergence theorems for some generalized nonexpansive mappings, J. Math. Anal. Appl. 340(2008) 1088-10995. https://doi.org/10.1016/j.jmaa. 2007.09.023.

[39] S. Thianwan, Common fixed points of new iterations for two asymptotically nonexpansive nonself-mappings in a Banach space. J. Comput. Appl. Math. 224(2009) 688-695. https://doi.org/10.1016/j . cam. 2008. 05.051.

[40] D. Thakur, B. S. Thakur, M. Postolache, A new iterative scheme for numerical reckoning fixed points of Suzuki's generalized nonexpansive mappings, Appl. Math. Comput. 275 (2016), 147-155. https://doi. org $/ 10.1016 / j$. amc. 2015.11.065.

[41] B. S. Thakur, D. Thakur, M. Postolache, A new iterative scheme for numerical reckoning fixed points of Suzuki's generalized nonexpansive mappings, Appl. Math. Comput. 275 (2016), 147-155.

[42] K. Ullah, M. Arshad, New iteration process and numerical reckoning fixed points in Banach spaces, Univ. Pol. Bucharest Sci. Bull. Ser. A, 79 (2017), 113-122.

[43] K. Ullah, M. Arshad, Numerical reckoning fixed points for Suzuki's generalized nonexpansive mappings via new iteration process, Filomat, 32(2018) 187-196. https://doi.org/10.2298/FIL1801187U.

[44] K. Ullah, M. Arshad, New three-step iteration process and fixed point approximation in Banach spaces, J. Linear Topol. Alg. 7(2) (2018) 87-100.

[45] K. Ullah, F. Ayaz, J. Ahmad, Some convergence results of M iterative process in Banach spaces, Asian-Eur. J. Math. 14 (2021) 2150017. https://doi.org/10.1142/S1793557121500170.

[46] A.M. Wazwaz, A reliable treatment for mixed Volterra-Fredholm integral equations, Appl. Math. Comput. 127 (2002) 405-414. https://doi.org/10.1016/S0096-3003(01)00020-0.

[47] T. Zamfirescu, Fixed point theorems in metric spaces, Arch. Math. (Basel). 23 (1972), 292-298. 